\title{
The Reliability of Fibro-test in Staging Orthotopic Liver Transplant Recipients with Recurrent Hepatitis C
}

\author{
Panagiotis Trilianos, Adamantios Tsangaris, Augustine Tawadros, Vrushak Deshpande \\ and Nikolaos Pyrsopoulos*
}

Division of Gastroenterology \& Hepatology, University Hospital, Rutgers - New Jersey Medical School, NJ, USA

\begin{abstract}
Background and Aims: Liver biopsy remains the gold standard for staging of chronic liver disease following orthotopic liver transplantation. Noninvasive assessment of fibrosis with Fibrotest (FT) is well-studied in immunocompetent populations with chronic hepatitis $C$ virus infection. The aim of this study is to investigate the diagnostic value of FT in the assessment of hepatic fibrosis in the allografts of liver transplant recipients with evidence of recurrent hepatitis C. Methods: We retrospectively compared liver biopsies and FT performed within a median of 1 month of each other in orthotopic liver transplantation recipients with recurrent hepatitis $C$. Results: The study population comprised 22 patients, most of them male (19/22), and with median age of 62 years. For all patients, there was at least a one-stage difference in fibrosis as assessed by liver biopsy compared to $\mathrm{FT}$, while for the majority $(16 / 22)$ there was at least a two-stage difference. The absence of correlation between the two modalities was statistically demonstrated (Mann-Whitney $U$ test, $p=0.01$ ). In detecting significant fibrosis (a METAVIR stage of F2 and above), an FT cut-off of 0.5 showed moderate sensitivity $(77 \%)$ and negative predictive value $(80 \%)$, but suboptimal specificity $(61 \%)$ and positive predictive value (58\%). Conclusions: In post-transplant patients with recurrent hepatitis C, FT appears to be inaccurately assessing the degree of allograft fibrosis, therefore limiting its reliability as a staging tool.

Citation of this article: Trilianos $P$, Tsangaris $A$, Tawadros $A$, Deshpande V, Pyrsopoulos $N$. The reliability of fibro-test in staging orthotopic liver transplant recipients with recurrent hepatitis C. J Clin Transl Hepatol 2020;8(1):9-12. doi: 10.14218/JCTH.2019.00038.
\end{abstract}

\section{Introduction}

Allograft re-infection by the hepatitis $\mathrm{C}$ virus (HCV) in treatment-naïve or unsuccessfully treated orthotopic liver transplant (OLT) recipients is swift and universal and used to

Keywords: Fibro-test; Orthotopic liver transplantation; Fibrosis; Recurrent hepatitis C; Hepatitis $C$ virus.

Abbreviations: DAA, direct-acting antiviral; FT, Fibro-Test; $\mathrm{HCV}$, hepatitis $\mathrm{C}$ virus; LB, liver biopsy; OLT, orthotopic liver transplant; RHC, recurrent hepatitis C; ROC, receiver operating characteristic; $V C T E$, vibration-controlled transient elastography. Received: 22 August 2019; Revised: 24 November 2019; Accepted: 24 December 2019

*Correspondence to: Nikolaos Pyrsopoulos, Division of Gastroenterology \& Hepatology, University Hospital, Rutgers - New Jersey Medical School, Medical Science Building, Room H-536, 185 S. Orange Ave, Newark, NJ 07103, USA. Tel: +1-973972-5252, Fax: +1-973-972-3144, E-mail: pyrsopni@njms.rutgers.edu be associated with an accelerated rate of fibrosis progression. ${ }^{1-3}$ The advent of direct-acting antivirals (DAAs) dramatically changed the landscape of HCV treatment, with safe and effective regimens that have high cure rates leading to significantly improved outcomes in the post-OLT setting ${ }^{4,5}$ Still, treatment of recurrent hepatitis $\mathrm{C}$ (RHC) in liver transplant recipients may pose a challenge. ${ }^{6}$ As such, there remains the necessity to periodically restage the relatively few patients who either fail or are unable to tolerate DAA treatment.

Liver biopsy (LB) is considered the gold standard for assessment of hepatic fibrosis, although imperfect. Sample size and quality factor greatly into diagnostic accuracy, ${ }^{7}$ and there is considerable inter- and intra-observer variability in the interpretation of histologic findings. ${ }^{8}$ As a procedure, it is infrequently associated with both major and minor complications but does carry some mortality risk. ${ }^{9,10}$ Protocol biopsies, traditionally employed in the post-transplant setting for surveillance of allograft integrity and function, are currently largely abandoned. ${ }^{11}$

Noninvasive assessment of fibrosis through serum markers, various types of elastography, imaging or combinations thereof mitigates the procedural risks associated with LB, making it a very attractive concept. Reliable noninvasive testing is currently accessible to aid in the care of patients with chronic HCV infection. Such is Fibro-test (FT), a proprietary algorithm comprising both direct and indirect markers of fibrosis, that has been well-studied and validated as a diagnostic tool in immunocompetent patients with viral hepatitis among other common chronic liver diseases. ${ }^{12,13}$

This study was designed to evaluate FT in the assessment of hepatic fibrosis in liver transplant recipients with RHC.

\section{Methods}

Retrospective review of charts of adult OLT recipients followed in our institution between the years of 2003 and 2016 was conducted. Criteria for enrollment included patients transplanted for end-stage liver disease due to chronic hepatitis $\mathrm{C}$ infection, with recurrence of viremia in the post-transplant period associated with histologically typical hepatic necroinflammation on LB (obtained for staging purposes), in the absence of a concurrent cause of liver injury, such as cellular rejection, hepato-vascular complications, biliary strictures, and de novo liver disease (e.g., nonalcoholic fatty liver disease or autoimmune hepatitis). Patients with moderate hepatitis (alanine aminotransferase $>200 \mathrm{IU} / \mathrm{mL}$ ), significant cholestasis (alkaline phosphatase or gamma-glutamyl transferase $>1.5 x$ the upper limit of normal), decompensated liver 
disease (defined as total bilirubin $>2.0 x$ the upper limit of normal and international normalized ratio $>1.5$ or the presence of ascites, hepatic encephalopathy, variceal bleeding) were excluded. Within those parameters, patients with fibrosing cholestatic hepatitis $C$ were naturally excluded. Any alcohol consumption was also an exclusion criterion. Patients must have had FT within 4 months of LB and either before or well after any antiviral treatment.

Demographic data (age, gender, and race) were recorded. All qualitative variables were expressed as frequencies and all quantitative as median values. Time elapsed from OLT to LB to FT was calculated. All biopsies were reviewed anew by an experienced hepato-pathologist and staged according to the METAVIR classification system. The size of the histologic specimen was documented. Fibro Test was acquired through the commercially available assay (FibroSURE; LabCorp, Burlington, NC, USA), which comprises a2-macroglobulin, haptoglobin, apo-lipoprotein A1, total bilirubin, and gamma-glutamyl transferase, while the addition of alanine aminotransferase offers a surrogate marker of hepatic necroinflammation (Acti-Test). ${ }^{14}$

Non-parametric testing (Mann-Whitney $U$ test) was implemented to compare LB to FT results. The sensitivity, specificity, and positive and negative predictive values of FT for any or significant fibrosis (as defined by a METAVIR score of F2 and above) were calculated. The diagnostic value of FT for the detection of significant fibrosis was further assessed by plotting the receiver operating curve (ROC). All statistical analyses were performed with SPSS version 24 (IBM Corp, Armonk, NY, USA).

\section{Results}

A total of 22 patients met the study criteria, including 19 men and 3 women, mostly Caucasian (54\%), with median age of 62 years. Infection with HCV genotype 1 was most prevalent $(18 / 22,81.8 \%)$. The most frequently used immunosuppressive agents were mycophenolate mofetil $(82 \%)$ and tacrolimus $(77 \%)$, often in combination (64\%). Median time elapsed from OLT to LB was 45 months, while median chronological distance of FT from LB was 1 month. Median length of biopsy specimens acquired was $15 \mathrm{~mm}$. More than one-third of the study population had no histologic evidence of fibrosis $(9 / 22,41 \%)$. Mild fibrosis was seen in 8 patients $(18 \%)$, moderate fibrosis in $8(36 \%)$, and 1 patient had advanced fibrosis (5\%). No patient was cirrhotic per LB.

There was at least a one-stage difference in fibrosis stage as assessed by LB versus that of FT in all patients; a two-stage difference was noted in 16 patients (73\%), and in 4 patients LB and FT were grossly discrepant. Mann-Whitney $U$ test further confirmed the discrepancy between modalities $(p=0.01)$. In detecting any fibrosis (METAVIR F1 and above), FT was $100 \%$ sensitive. In detecting significant fibrosis (METAVIR F2 and above), an FT cut-off of 0.5 showed a sensitivity of $77 \%$, a specificity of $61 \%$, a positive predictive value of $58 \%$ and negative predictive value of $80 \%$. A ROC curve was plotted, reflecting the overall performance (Fig. 1). As we only had one patient with biopsy-proven advanced fibrosis and none with cirrhosis, we deferred measuring the respective values for detecting advanced stages of liver disease.

\section{Discussion}

Historical evidence demonstrates that the course of hepatitis C tends to be more accelerated following liver transplantation, with histologic hepatitis found in the majority of allografts

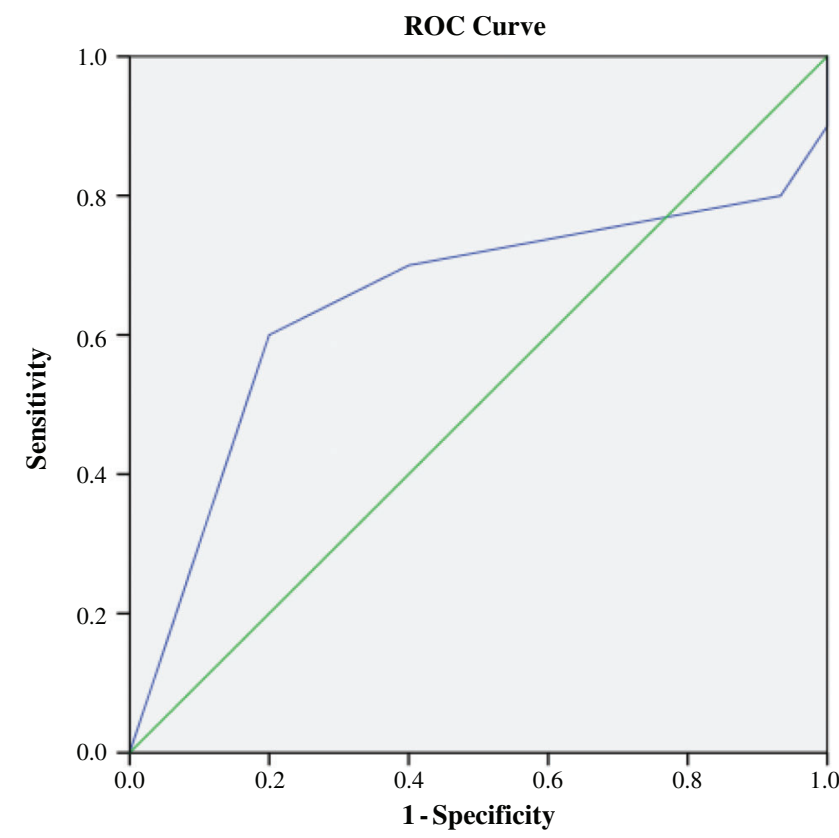

Fig. 1. Diagnostic accuracy of FT as determined by area under the curv $($ AUROC $=0.647)$.

within 1 year and cirrhosis developing in up to $40 \%$ of patients within 5 years. ${ }^{1-3}$ There is no denying that the use of DAAs in liver recipients with $\mathrm{RHC}$ or even kidney recipients with de novo HCV infection has largely mitigated this problem, enabling timely, safe and cost-effective viral eradication. ${ }^{4,5,15}$ However, data on the utilization of DAAs post-transplant are limited when compared to the abundance of large and small, prospective and retrospective studies in immunocompetent patients. The introduction of all-oral, interferon-free DAAs into routine clinical practice is relatively recent (sofosbuvir/ ledipasvir attained Federal Drug Administration approval in October of 2014, with others following suit), and as such there is paucity of robust data on the SVR rates in DAA- and more specifically the NS5A-experienced OLT recipients with RHC. This is reflected in large clinical trials, where participants were either treatment-naïve or had received interferon- or sofosbuvir-based regimens. ${ }^{16-20}$ The majority were also genotype-1 infected. ${ }^{15-17,20}$ The issues of drug-drug interactions, varying degrees of concomitant renal insufficiency, and rarely acute rejection also come into consideration in the post-transplant setting, making the treatment of RHC more challenging. ${ }^{6,17,21}$ What is more, sustained virological response rates appear to be lower in simultaneous liver-kidney transplant recipients. ${ }^{17}$ As such, until a universally accepted, streamlined, $100 \%$ effective and caveat-free regimen is found to treat RHC in liver recipients, there will be a need to monitor disease progression in those few who fail or do not tolerate our current options.

Liver biopsy is still accepted as the definitive method to stage liver disease in the pre- and post-OLT setting, despite its well-documented shortcomings. ${ }^{7,9,10}$ Protocol biopsies are no longer the norm, although they do have proponents. ${ }^{11}$ Noninvasive assessment of fibrosis through (direct or indirect) serum markers or elastographic techniques (with or without concurrent imaging) has been gaining traction over the last 15 years. Simple, point-of-care clinical tools have been 
extensively studied and validated in viral hepatitis. ${ }^{22,23}$ Among proprietary tests, the Fibro Test (BioPredictive Paris, France) is now widely used in clinical practice as part of the pretreatment evaluation of HCV infection. ${ }^{12,13,24,25}$ Moreover, FT is well-validated in immunocompetent HCV-infected patients, ${ }^{13,26}$ but the data in the post-transplant population are decidedly mixed.

The largest study to research the utility of FT in the posttransplant setting was the one by Beckebaum et al., ${ }^{27} \mathrm{com}-$ prising a population of 157 OLT recipients, a third of whom had RHC. In the HCV group, FT performed best, yielding an area under the ROC curve of 0.79 for the diagnosis of advanced fibrosis and 0.81 for cirrhosis, and it was less accurate in the non-HCV group, with area under the ROC curve values of 0.70 and 0.75 respectively. Of note, the diagnostic value of FT in detecting moderate necroinflammation (grade A2 and above) in patients with RHC appeared to be very limited, with an area under the ROC curve of only 0.60 . In contrast, vibration-controlled transient elastography (VCTE) was found to be the most accurate noninvasive test in establishing advanced fibrosis and cirrhosis in both HCV and nonHCV OLT recipients.

Earlier, the Bologna Liver Transplantation Group ${ }^{28}$ had also evaluated the utility serologic markers (among them, FT) and VCTE in detecting significant fibrosis (defined as a METAVIR score of $\mathrm{F} 2$ and above) in patients with RHC following liver transplantation. FT performed extremely poorly in this setting, with a cut-off of 0.8 yielding a sensitivity of $56 \%$ and a specificity of $61 \%$, and an overall area under the ROC curve of 0.56 . Elastography performed excellently and was found to be superior to other noninvasive testing procedures (area under the ROC curve of 0.94). Notably, parameters to calculate the FT score were available for only a subgroup of the total population (36 out of 56 patients) and only a minority of those had advanced fibrosis or cirrhosis $(n=5)$; therefore, conclusions on the diagnostic value of FT in detecting late stages of fibrosis cannot be drawn from this study.

The accuracy of FT has also been investigated in the setting of renal transplantation. In a 2009 French study 29 including $26 \mathrm{HCV}$-infected kidney recipients, FT correctly classified $80 \%$ of patients with no or mild fibrosis (F0-F1), performing similarly to transient elastography. However, the sensitivity and accuracy of FT in detecting advanced fibrosis or cirrhosis were rather dismal (sensitivity of $\sim 31 \%$, area under the ROC curve of 0.55 ).

A 2010 meta-analysis by Cholongitas et al. ${ }^{30}$ found VCTE to be superior to serologic markers, including FT, in the assessment of significant fibrosis and cirrhosis in OLT recipients with RHC; although, it should be noted that only one study ${ }^{28}$ evaluating FT was included. Most recently, the ability of FT to discriminate between the presence and absence of significant fibrosis (as defined by an Ishak score of $>3$ ) was investigated in an Italian study including 51 liver recipients with $\mathrm{RHC}^{31}$ a FT cut-off value of 0.716 had a positive predictive value of $58 \%$ but a negative predictive value of almost $94 \%$ for significant fibrosis, while the overall accuracy of FT (area under the ROC curve of 0.848 ) was second only to acoustic radiation forced imaging and superior to all other chemistry-based indices. Combination of both acoustic radiation forced imaging and FT appeared to offer no additional benefit.

Our investigation reached a negative conclusion regarding the utility of FT in liver recipients with RHC. In a cohort of 22 OLT recipients with RHC, FT was able to detect the presence of any fibrosis but consistently and oftentimes grossly overestimated its degree. We applied strict selection criteria to reasonably exclude patients with co-existing nonHCV-related liver injury or severe hepatic necroinflammation, either of which would confound the interpretation of FT. By the same token, the risk of selection bias is acknowledged. The mean chronological distance between FT and biopsy was 1.5 months, making their acquisition practically concurrent and therefore eliminating the possibility of any meaningful fibrosis progression in the time elapsed from one to the other. Fibro Test was also obtained either prior to or well after antiviral treatment, as improvement of biochemical parameters during treatment could also potentially affect its reliability. The small number of patients is our foremost limitation. Among them, only one had biopsy-proven advanced fibrosis and none had cirrhosis. Therefore, the utility of FT in detecting advanced stages of liver disease in post-transplant patients with RHC could not be assessed in the present study.

\section{Conclusions}

Contrary to immunocompetent HCV-infected patients, FT appears to significantly over-stage OLT recipients with RHC and therefore its diagnostic value as a non-invasive test for the assessment of fibrosis is questionable. This may be explained by the reduction of apo-lipoprotein A1 levels, a component of FT, by calcineurin inhibitors. ${ }^{32,33}$ Similar to the findings in immunocompetent patients, elastography appears to be superior, ${ }^{28-30,34,35}$ though a universally accepted noninvasive strategy to detect hepatic fibrosis in the post-transplant setting has yet to be established.

\section{Funding}

None to declare.

\section{Conflict of interest}

Nikolaos Pyrsopoulos is a recipient of research grants from Gilead, Abbvie, Merck and Roche. The other authors have no conflict of interests related to this publication.

\section{Author contributions}

Study concept and design (NP, VD), data collection (AdT, AuT), analysis and interpretation of data (PT, AdT), drafting of manuscript (PT), critical revision, study supervision (NP).

\section{References}

[1] Gane E. The natural history and outcome of liver transplantation in hepatitis C virus-infected recipients. Liver Transpl 2003;9:S28-S34. doi: 10. $1053 /$ jlts.2003.50248.

[2] Forman LM, Lewis JD, Berlin JA, Feldman HI, Lucey MR. The association between hepatitis $C$ infection and survival after orthotopic liver transplantation. Gastroenterology 2002;122:889-896. doi: 10.1053/gast.2002.32418.

[3] Berenguer M, Prieto M, Rayón JM, Mora J, Pastor M, Ortiz V, et al. Natural history of clinically compensated hepatitis $C$ virus-related graft cirrhosis after liver transplantation. Hepatology 2000;32:852-858. doi: 10.1053/jhep. 2000.17924

[4] Cotter TG, Paul S, Sandıkçı B, Couri T, Bodzin AS, Little EC, et al. Improved graft survival after liver transplantation for recipients with hepatitis $C$ virus in the direct-acting antiviral era. Liver Transpl 2019;25:598-609. doi: 10 . $1002 /$ It. 25424

[5] Belli LS, Perricone G, Adam R, Cortesi PA, Strazzabosco M, Facchetti R, et al. Impact of DAAs on liver transplantation: Major effects on the evolution of 
indications and results. An ELITA study based on the ELTR registry. J Hepatol 2018;69:810-817. doi: 10.1016/j.jhep.2018.06.010.

[6] Saab S, Rheem J, Jimenez M, Bau S, Choi G, Durazo F, et al. Curing hepatitis C in liver transplant recipients is associated with changes in immunosuppressant use. J Clin Transl Hepatol 2016;4:32-38. doi: 10.14218/JCTH.2016.00001.

[7] Bedossa $P$, Dargère $D$, Paradis V. Sampling variability of liver fibrosis in chronic hepatitis C. Hepatology 2003;38:1449-1457. doi: 10.1016/j.hep. 2003.09.022.

[8] Poynard T, Munteanu M, Imbert-Bismut F, Charlotte F, Thabut D, Le Calvez S, et al. Prospective analysis of discordant results between biochemical markers and biopsy in patients with chronic hepatitis C. Clin Chem 2004;50:13441355. doi: $10.1373 /$ clinchem.2004.032227.

[9] Piccinino F, Sagnelli E, Pasquale G, Giusti G. Complications following percutaneous liver biopsy. A multicentre retrospective study on 68,276 biopsies. J Hepatol 1986;2:165-173. doi: 10.1016/s0168-8278(86)80075-7.

[10] Seeff LB, Everson GT, Morgan TR, Curto TM, Lee WM, Ghany MG, et al. Complication rate of percutaneous liver biopsies among persons with advanced chronic liver disease in the HALT-C trial. Clin Gastroenterol Hepatol 2010;8: 877-883. doi: 10.1016/j.cgh.2010.03.025.

[11] Mells G, Mann C, Hubscher S, Neuberger J. Late protocol liver biopsies in the liver allograft: a neglected investigation? Liver Transpl 2009;15:931-938. doi: $10.1002 /$ lt.21781.

[12] Poynard T, Morra R, Halfon P, Castera L, Ratziu V, Imbert-Bismut F, et al. Meta-analyses of FibroTest diagnostic value in chronic liver disease. BMC Gastroenterol 2007;7:40. doi: 10.1186/1471-230X-7-40.

[13] Poynard T, Munteanu M, Deckmyn O, Ngo Y, Drane F, Castille JM, et al. Validation of liver fibrosis biomarker (FibroTest) for assessing liver fibrosis progression: proof of concept and first application in a large population. J Hepatol 2012;57:541-548. doi: 10.1016/j.jhep.2012.04.025.

[14] Poynard T, Munteanu M, Ngo Y, Castera L, Halfon P, Ratziu V, et al. ActiTest accuracy for the assessment of histological activity grades in patients with chronic hepatitis C, an overview using Obuchowski measure. Gastroentero Clin Biol 2010;34:388-396. doi: 10.1016/j.gcb.2010.05.001.

[15] Axelrod DA, Schnitzler MA, Alhamad T, Gordon F, Bloom RD, Hess GP, et al. The impact of direct-acting antiviral agents on liver and kidney transplant costs and outcomes. Am J Transplant 2018;18:2473-2482. doi: 10. 1111/ajt.14895.

[16] Charlton M, Everson GT, Flamm SL, Kumar P, Landis C, Brown RS Jr, et al. Ledipasvir and sofosbuvir plus ribavirin for treatment of HCV infection in patients with advanced liver disease. Gastroenterology 2015;149:649659. doi: 10.1053/j.gastro.2015.05.010.

[17] Saxena V, Khungar V, Verna EC, Levitsky J, Brown RS Jr, Hassan MA, et al. Safety and efficacy of current direct-acting antiviral regimens in kidney and liver transplant recipients with hepatitis C: Results from the HCV-TARGET study. Hepatology 2017;66:1090-1101. doi: 10.1002/hep.29258.

[18] Coilly A, Fougerou-Leurent C, de Ledinghen V, Houssel-Debry P, Duvoux C, D Martino $\mathrm{V}$, et al. Multicentre experience using daclatasvir and sofosbuvir to treat hepatitis C recurrence - The ANRS CUPILT study. J Hepatol 2016;65: 711-718. doi: 10.1016/j.jhep.2016.05.039.

[19] Reau N, Kwo PY, Rhee S, Brown RS Jr, Agarwal K, Angus P, et al. Glecaprevir/pibrentasvir treatment in liver or kidney transplant patients with hepatitis C virus infection. Hepatology 2018;68:1298-1307. doi: 10.1002/hep. 30046.

[20] Poordad F, Schiff ER, Vierling JM, Landis C, Fontana RJ, Yang R, et al. Daclatasvir with sofosbuvir and ribavirin for hepatitis $C$ virus infection with advanced cirrhosis or post-liver transplantation recurrence. Hepatology 2016;63:1493-1505. doi: 10.1002/hep.28446.
[21] Smolders EJ, Jansen AME, Ter Horst PGJ, Rockstroh J, Back DJ, Burger DM. Viral hepatitis $C$ therapy: Pharmacokinetic and pharmacodynamic considerations: A 2019 update. Clin Pharmacokinet 2019;58:1237-1263. doi: 10. 1007/s40262-019-00774-0.

[22] Chou R, Wasson N. Blood tests to diagnose fibrosis or cirrhosis in patients with chronic hepatitis $C$ virus infection: a systematic review. Ann Intern Med 2013;158:807-820. doi: 10.7326/0003-4819-158-11-201306040-00005.

[23] Kim BK, Kim DY, Park JY, Ahn SH, Chon CY, Kim JK, et al. Validation of FIB-4 and comparison with other simple noninvasive indices for predicting live fibrosis and cirrhosis in hepatitis B virus-infected patients. Liver Int 2010 ; 30:546-553. doi: 10.1111/j.1478-3231.2009.02192.x.

[24] Poynard T, Imbert-Bismut F, Munteanu M, Messous D, Myers RP, Thabut $D$, et al. Overview of the diagnostic value of biochemical markers of liver fibrosis (FibroTest, HCV FibroSure) and necrosis (ActiTest) in patients with chronic hepatitis C. Comp Hepatol 2004;3:8. doi: 10.1186/1476-5926-3-8.

[25] Munteanu M, Tiniakos D, Anstee $O$, Charlotte F, Marchesini G, Bugianesi $E$, et al. Diagnostic performance of FibroTest, SteatoTest and ActiTest in patients with NAFLD using the SAF score as histological reference. Aliment Pharmacol Ther 2016;44:877-889. doi: 10.1111/apt.13770.

[26] Zarski JP, Sturm N, Guechot J, Paris A, Zafrani ES, Asselah T, et al. Comparison of nine blood tests and transient elastography for liver fibrosis in chronic hepatitis C: the ANRS HCEP-23 study. J Hepatol 2012;56:55-62. doi: 10 1016/j.jhep.2011.05.024.

[27] Beckebaum S, Iacob S, Klein CG, Dechêne A, Varghese J, Baba HA, et al. Assessment of allograft fibrosis by transient elastography and noninvasive biomarker scoring systems in liver transplant patients. Transplantation 2010;89:983-993. doi: 10.1097/TP.0b013e3181cc66ca.

[28] Corradi F, Piscaglia F, Flori S, D'Errico-Grigioni A, Vasuri F, Tamé MR, et al. Assessment of liver fibrosis in transplant recipients with recurrent HCV infection: usefulness of transient elastography. Dig Liver Dis 2009;41:217-225. doi: $10.1016 / j$.dld.2008.06.009.

[29] Alric L, Kamar N, Bonnet D, Danjoux M, Abravanel F, Lauwers-Cances V, et al. Comparison of liver stiffness, fibrotest and liver biopsy for assessment of liver fibrosis in kidney-transplant patients with chronic viral hepatitis. Transpl Int 2009:22:568-573 doi: 10.1111/j.1432-2277.2009.00834.

[30] Cholongitas E, Tsochatzis E, Goulis ], Burroughs AK. Noninvasive tests for evaluation of fibrosis in HCV recurrence after liver transplantation: a systematic review. Transpl Int 2010;23:861-870. doi: 10.1111/j.1432-2277.2010. 01142.x.

[31] Bignulin S, Falleti E, Cmet S, Cappello D, Cussigh A, Lenisa I, et al. Usefulness of acoustic radiation force impulse and fibrotest in liver fibrosis assess ment after liver transplant. Ann Hepatol 2016;15:200-206. doi: 10 5604/16652681.1193710.

[32] Chakkera HA, Mandarino LJ. Calcineurin inhibition and new-onset diabetes mellitus after transplantation. Transplantation 2013;95:647-652. doi: 10 1097/TP.0b013e31826e592e.

[33] Chakkera HA, Kudva Y, Kaplan B. Calcineurin inhibitors: Pharmacologic mechanisms impacting both insulin resistance and insulin secretion leading to glucose dysregulation and diabetes mellitus. Clin Pharmacol Ther 2017; 101:114-120. doi: 10.1002/cpt.546.

[34] Bhat M, Tazari M, Sebastiani G. Performance of transient elastography and serum fibrosis biomarkers for non-invasive evaluation of recurrent fibrosis after liver transplantation: A meta-analysis. PLoS One 2017;12:e0185192. doi: 10.1371/journal.pone.0185192.

[35] Carrión JA, Navasa M, Bosch J, Bruguera M, Gilabert R, Forns X. Transient elastography for diagnosis of advanced fibrosis and portal hypertension in patients with hepatitis $C$ recurrence after liver transplantation. Liver Transp 2006;12:1791-1798, doi: 10.1002/lt.20857. 